\title{
MORFOGÉNESIS DE LA FASE SEXUAL DE SIETE TAXA EPÍFITOS DE POLYPODIUM (POLYPODIACEAE) DE MÉXICO
}

\author{
Nancy Vázquez-Pérez, Aniceto Mendoza-Ruiz y Blanca Pérez-García \\ Universidad Autónoma Metropolitana, Iztapalapa, Departamento de Biología,Área \\ de Botánica Estructural y Sistemática Vegetal, Avenida San Rafael Atlixco 186, \\ Colonia Vicentina, 09340 México, D.F., México. \\ 1Autor para la correspondencia: amr@xanum.uam.mx
}

\section{RESUMEN}

Se analiza y describe la morfología de la fase sexual de siete taxa epífitos de Polypodium que crecen en México. Las esporas son monoletes, elipsoidales a reniformes, con el perisporio verrucado a tuberculado. El patrón de germinación corresponde al tipo Vittaria para todas las especies; en cuanto al desarrollo protálico, se observó el tipoDrynaria en Polypodium furfuraceum, P. plebeium, P. polypodioides var. aciculare, $P$. polypodioides var. polypodioides y $P$. rhodopleuron y el tipo Aspidium en $P$. fraternum y $P$. lepidotrichum. Con respecto a los gametangios, en algunas especies se formaron gametofitos unisexuales y/o bisexuales. Las especies que desarrollaron protalos con arquegonios fueron $P$. fraternum, $P$. furfuraceum, $P$. lepidotrichum, $P$. plebeium, $P$. polypodioides var. aciculare y $P$. polypodioides var. polypodioides. Los anteridios se originaron en $P$. rhodopleuron, $P$. plebeium y $P$. polypodioides var. polypodioides. Se observaron yemas para la propagación vegetativa en los gametofitos de P. lepidotrichum y P. rhodopleuron.

Palabras clave: esporas, gametangios, gametofitos, germinación, helechos epífitos.

\section{ABSTRACT}

Morphology of the sexual phase of seven epiphytic taxa of Polypodium that grow in Mexico was observed and described. The spores of all species studied are monolete, ellipsoid to reniform, and verrucate to tuberculate. All the species analyzed have the Vittaria-type pattern of germination. In regard to the prothallial development, the Drynaria-type was observed in Polypodium furfuraceum, P. plebeium, P. polypodioides var. aciculare, P. polypodioides 
var. polypodioides, whereas $P$. rhodopleuron. $P$. fraternum and $P$. lepidotrichum displayed the Aspidium-type pattern. Unisexual and/or bisexual gametophytes were observed in some species. Polypodium fraternum, P. furfuraceum, P. lepidotrichum, P. plebeium, $P$. polypodioides var. aciculare and $P$. polypodioides var. polypodioides developed archegonial gametophytes while $P$. rhodopleuron, $P$. plebeium and $P$. polypodioides var. polypodioides formed antheridial gametophytes. A trend to the vegetative propagation of the gametophytes was observed in P. lepidotrichum and P. rhodopleuron.

Key words: epiphytic ferns, gametangia, gametophytes, germination, spores.

\section{INTRODUCCIÓN}

La familia Polypodiaceae, incluye alrededor de 40 géneros y aproximadamente 600 especies de amplia distribución (Moran y Riba, 1995). Entre los primeros se encuentra Polypodium, el cual concentra unas 120 especies, en su mayoría epífitas y de distribución americana. Sus esporofitos poseen rizomas cortos a largamente rastreros y escamosos; pecíolo y raquis sulcados del lado adaxial; hojas con la lámina pinnatisecta a pinnada; soros redondos u ocasionalmente elípticos, sin indusio, dispuestos en los ápices de las nervaduras; cápsulas esporangiales glabras o setulosas; esporas amarillas, monoletes, elipsoidales a reniformes (Moran y Riba, 1995; Mickel y Smith, 2004).

La circunscripción taxonómica de Polypodium es problemática, señalándose en distintos trabajos la necesidad de revisarla (Smith et al., 2006). Actualmente varios autores reconocen de manera informal dentro del género distintos complejos de especies. México es considerado como centro de diversificación de algunos de ellos como por ejemplo los de Polypodium dulce, P. plesiosorum y P. polypodioides (Moran y Riba, 1995; Mickel y Smith, 2004).

En los helechos, una gran parte del conocimiento biológico que se tiene del grupo se ha generado a partir de la fase esporofítica, sin embargo, son diversos los autores que han señalado la importancia que tiene el estudio de la gametofítica. Así por ejemplo, Nayar y Kaur $(1969,1971)$ junto con Atkinson (1973) han señalado que la morfología de los protalos, en combinación con los caracteres morfológicos del esporofito, puede ser útil para caracterizar taxonómicamente a las especies de pteridofitas.

En lo que se refiere a Polypodium en particular, distintos autores han realizado estudios bioquímicos y fisiológicos en los gametofitos de algunas especies (Knudson, 1940; Smith y Robinson 1969, 1971, 1975; Smith, 1972; Smith et al., 1973; Roy, 
1987; Reixach et al., 1996; Muccifora, 2008); también se ha analizado la influencia de la luz visible y de los rayos X como inhibidores de la germinación, crecimiento y diferenciación de los mismos (Spies y Krouk, 1977; Agnew et al., 1984, 1985; Roy, 1987; Ranal, 1991), así como el efecto de la densidad poblacional en su desarrollo (Smith y Rogan, 1970).

En cuanto al estudio de la morfogénesis de la fase sexual en el género, destacan varias contribuciones en las que se describe la polaridad de la espora, el inicio de la germinación, el desarrollo de los rizoides, el patrón de germinación, los tipos de desarrollo protálico y la diferenciación sexual y reproducción de Polypodium aureum L., P. australe (Fée) Maxon, P. cambricum L., P. chnoodes Spreng., P. feuillei Bertero, $P$. hirsutissimum Raddi, $P$. irioides Poir., $P$. lepidotrichum (Fée) Maxon, $P$. pectinatum L., P. pellucidum Kaulf., P. plumula Humb. \& Bonpl. ex Willd, P. verrucosum Hook., P. virginianum L., P. vulgare L. y P. vulgare L. var. occidentale Hook (Wuist, 1916; Steil, 1921; Pickett y Thayer, 1927; Hartman, 1931; Davie, 1951, 1978; Stokey, 1951, 1959; Nayar y Kachroo, 1953; Nayar, 1955, 1962; Atkinson y Stokey, 1964, 1970; Nayar y Kaur, 1968, 1969, 1971; Nayar y Raza, 1970; Atkinson, 1973; Kott y Peterson, 1974; Raghavan, 1974, 1980, 1985 y 1989; Welling y Haufler, 1993; Reyes-Jaramillo y Pérez-García, 1994; Shorina, 2001; Chiou y Farrar, 1997a, 1997b; van Uffelen, 1997; Gabriel y Galán et al., 2008).

En el presente trabajo se estudia la fase sexual de siete taxa epífitos de Polypodium (Polypodiaceae): Polypodium fraternum Schltdl. et Cham. y P. rhodopleuron Kunze, que se circunscriben dentro del complejo de Polypodium plesiosorum, y P. furfuraceum Schltdl. et Cham., P. lepidotrichum (Fée) Maxon, P. plebeium Schltdl. et Cham., P. polypodioides (L.) Watt var. aciculare Weath. y P. polypodioides (L.) Watt var. polypodioides que se incluyen en el complejo de $P$. polypodioides, proporcionando información sobre las características de la espora y su tipo de germinación, el tipo de desarrollo protálico, la morfología del gametofito adulto y de los gametangios. De esta manera se pretende contribuir al conocimiento de la fase sexual de las pteridofitas tropicales americanas.

\section{MATERIAL Y MÉTODOS}

Las esporas de las especies en estudio se obtuvieron de hojas fértiles de diferentes individuos que fueron recolectados en distintas localidades del estado de Hidalgo (Cuadro 1); las pinnas con soros se colocaron en sobres de papel y se dejaron secar a temperatura ambiente por una semana; los ejemplares de respaldo se 
Cuadro 1.- Localidades de las especies estudiadas. BE, Bosque de encino; BMM, Bosque mesófilo de montaña.

\begin{tabular}{|c|c|c|c|}
\hline Taxa & Número de colecta & Localidad & $\begin{array}{l}\text { Tipo de vege- } \\
\text { tación/ Altitud } \\
\text { (m s.n.m.) }\end{array}$ \\
\hline $\begin{array}{l}\text { P. fraternum } \\
\text { Schltdl. et Cham. }\end{array}$ & A. Mendoza 1440 & $\begin{array}{l}2 \text { km sobre la desviación a } \\
\text { Huayacocotla, a partir del cami- } \\
\text { no San Agustín Metzquititlán- } \\
\text { Zacualtipán, Mpio. San Agustín } \\
\text { Metzquititlán, Hidalgo. }\end{array}$ & $\mathrm{BE} / 2049$ \\
\hline $\begin{array}{l}\text { P. furfuraceum } \\
\text { Schltdl. et Cham. }\end{array}$ & N. Vázquez 2 & $\begin{array}{l}\text { Río Malila. Mpio. Molango de } \\
\text { Escamilla, Hidalgo. }\end{array}$ & BMM/1240 \\
\hline $\begin{array}{l}\text { P. lepidotrichum } \\
\text { (Fée) Maxon }\end{array}$ & A. Mendoza 1443 & $\begin{array}{l}\text { Predio Casas Viejas, Mpio. San } \\
\text { Agustín Metzquititlán, Hidalgo. }\end{array}$ & $\mathrm{BE} / 2049$ \\
\hline \multirow[t]{2}{*}{$\begin{array}{l}\text { P. plebeium Schltdl. } \\
\text { et Cham. }\end{array}$} & A. Mendoza 1433N. & $\begin{array}{l}1.8 \text { km sobre la desviación a } \\
\text { Zoquizoquipan, a partir de la } \\
\text { carretera Zacualtipán-Molango, } \\
\text { Mpio. Metztitlán, Hidalgo. }\end{array}$ & $\mathrm{BE} / 2049$ \\
\hline & Vázquez 3 & $\begin{array}{l}\text { Río Malila. Mpio. Molango de } \\
\text { Escamilla Hidalgo. }\end{array}$ & BMM/1240 \\
\hline $\begin{array}{l}\text { P. polypodioides (L.) } \\
\text { Watt var. aciculare } \\
\text { Weath. }\end{array}$ & N. Vázquez 9 & $\begin{array}{l}\text { Río Malila. Mpio. Molango de } \\
\text { Escamilla, Hidalgo. }\end{array}$ & BMM/1240 \\
\hline $\begin{array}{l}\text { P. polypodioides (L.) } \\
\text { Watt var. } \\
\text { polypodioides }\end{array}$ & A. Mendoza 1425 & $\begin{array}{l}4 \text { km después de Ahuecatlán } \\
\text { rumbo a Contepec, Mpio. } \\
\text { Calnari, Hidalgo. }\end{array}$ & $\mathrm{BE} / 2049$ \\
\hline $\begin{array}{l}\text { P. rhodopleuron } \\
\text { Kunze }\end{array}$ & $\begin{array}{l}\text { N. Vázquez } 1 \\
\text { N. Vázquez } 4 \\
\text { N. Vázquez } 7\end{array}$ & $\begin{array}{l}\text { Río Malila. Mpio. Molango de } \\
\text { Escamilla, Hidalgo. }\end{array}$ & BMM/1240 \\
\hline
\end{tabular}

identificaron y se encuentran depositados en el Herbario Metropolitano Ramón Riba y Nava (UAMIZ) de la Universidad Autónoma Metropolitana, Iztapalapa.

Una vez liberadas las esporas dentro de los sobres, se tamizaron con una malla metálica con poros de $0.074 \mathrm{~mm}$ de diámetro. Se midieron un total de 30 por especie para obtener los tamaños (mínimo, promedio y máximo). Posteriormente, éstas fueron esparcidas con un pincel de pocas cerdas sobre la superficie de una placa de agar enriquecido con medio de cultivo de Thompson, previamente 
esterilizado (Klekowski, 1969). Por cada taxon se sembraron cinco cajas de Petri de $5 \mathrm{~cm}$ de diámetro, de las cuales una se puso en la oscuridad para probar fotoblastismo.

Las cajas con esporas se guardaron dentro de bolsas de polietileno transparente y se colocaron en un estante tipo esqueleto, con un fotoperiodo de $12 \mathrm{hrs} \mathrm{luz} /$ oscuridad (lámparas Solar 75 Watts, luz de día) y a una temperatura de $22-26^{\circ} \mathrm{C}$ en condiciones de laboratorio.

Después de la siembra se revisaron diariamente los cultivos hasta que se produjo la germinación, posteriormente se hicieron observaciones de manera semanal, para agregarles agua y obtener los datos y fotomicrografías de las distintas etapas de desarrollo de los gametofitos, mismas que fueron tomadas con un microscopio óptico Olympus BX- 41 y una cámara fotográfica Olympus C-5060.

\section{RESULTADOS}

Esporas: Las especies estudiadas son homospóricas, monoletes, elipsoidales a reniformes y aclorofílicas, de color amarillo a pardo oscuro y la ornamentación del perisporio es verrucada a tuberculada en todos los taxa (Tryon y Tryon, 1982). En cuanto al tamaño de las esporas, las más pequeñas fueron las de Polypodium polypodioides var. aciculare con (31) 40 (46) $\mu \mathrm{m}$ de largo x (20) 28 (34) $\mu \mathrm{m}$ de ancho (mínimo, promedio, máximo) y P. polypodioides var. polypodioides (30) $40(57) \mu \mathrm{m}$ x (24) 30 (40) $\mu \mathrm{m}$, las más grandes corresponden a P. rhodopleuron (60) 70 (77) $\mu \mathrm{m}$ x (47) $54(60) \mu \mathrm{m}$, el resto de las especies presenta un tamaño intermedio: $P$. fraternum (46) 65 (52) $\mu \mathrm{m} \times$ (31) 47 (36) $\mu \mathrm{m}, P$. furfuraceum (48) 57 (69) $\mu \mathrm{m} \times$ (35) 44 (58) $\mu \mathrm{m}$, P. lepidotrichum (39) 46 (58) $\mu \mathrm{m} \times$ (27) 31 (38) $\mu \mathrm{m}$, P. plebeium (41) 51 (60) $\mu \mathrm{m}$ x (27) 33 (40) $\mu \mathrm{m}$ (Figs. 1-4).

Germinación: Las esporas germinan entre los 6 y los 8 días posteriores a la siembra; en este proceso se observan grandes glóbulos de aceite (gotas lipídicas) de color amarillo en la primera célula protálica y que seguramente participan en la división celular y el crecimiento del gametofito, característica que comparte con muchas otras Polypodiaceae s. str. (Ramírez y Pérez-García, 1998; Pérez-García et al., 2001; Reyes Jaramillo et al., 2003). El patrón de germinación que presentan es tipo-Vittaria, en el cual la célula rizoidal se origina a partir de una división perpendicular al eje polar de la espora y la formación de la primera célula protálica se define por una división perpendicular a la anterior originando dos células, una de ellas permanece en reposo y la otra se parte dando inicio al desarrollo del filamento germinativo. La 

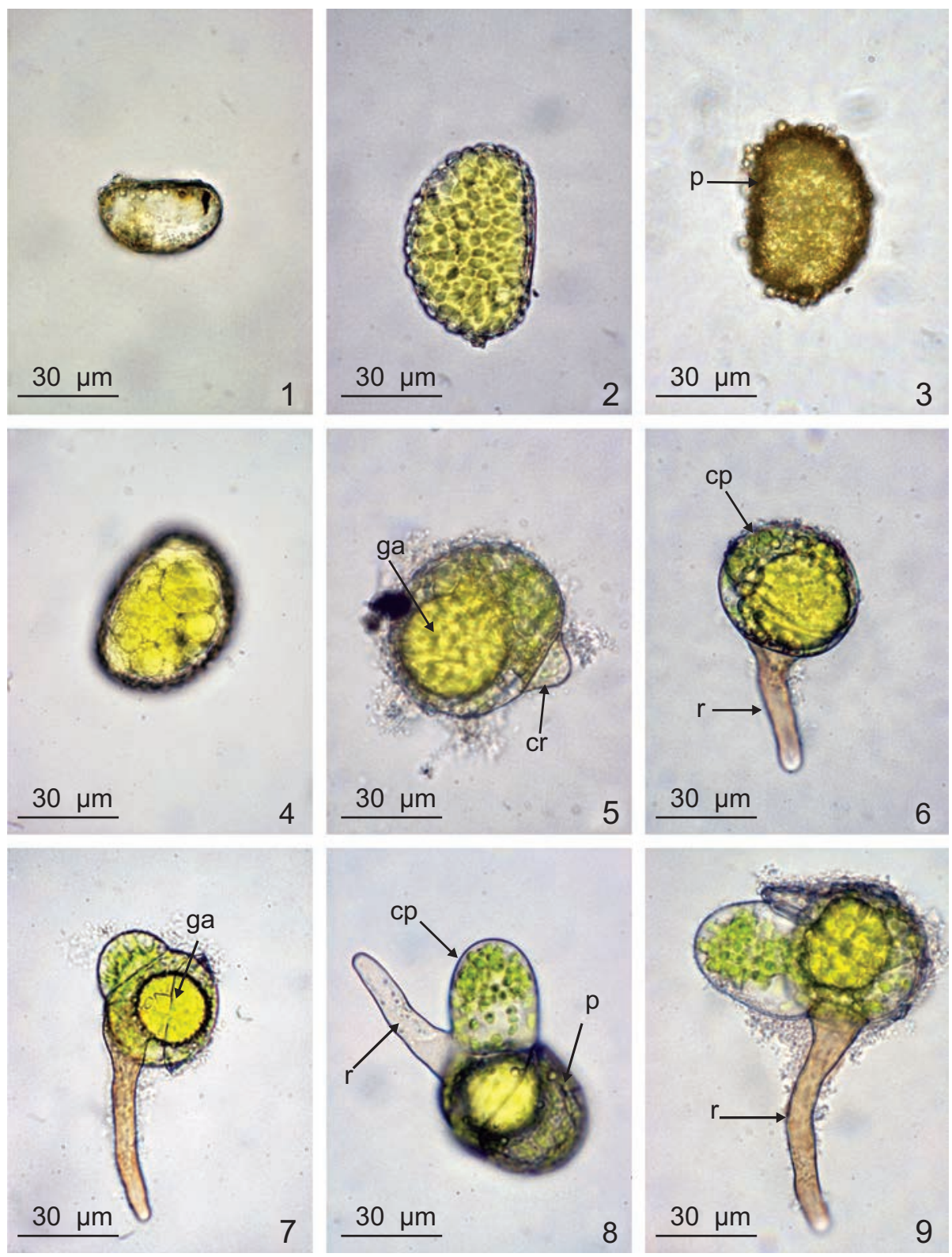

Figs. 1-4. Esporas de Polypodium, 1. Polypodium polypodioides var. aciculare, 2. P. plebeium, 3. P. lepidotrichum, 4. P. rhodopleuron, 5-9. Germinación de esporas en Polypodium, 5. P. plebeium (6 días), 6. P. polypodioides var. polypodioides (8 días), 7. P. polypodioides var. aciculare (8 días), 8. $P$. fraternum (8 días), 9. $P$. plebeium (6 días), $\mathrm{cp}=$ célula protálica, $\mathrm{cr}=$ célula rizoidal, ga $=$ glóbulo de aceite, $\mathrm{p}=$ perisporio, $\mathrm{r}=$ rizoide. 
célula rizoidal permanece indivisa y es hialina, mientras que las protálicas presentan abundantes cloroplastos (Figs. 5-9).

Fase filamentosa: Después de la germinación, por divisiones transversales se origina un filamento delgado y uniseriado de 3 a 8 células protálicas de longitud, con numerosos cloroplastos y glóbulos de aceite de color amarillo en la célula protálica inicial, esta fase es efímera, ocurre entre los 10 y los 20 días en promedio para todas las especies (Figs. 10-12).

Fase laminar: En Polypodium furfuraceum, P. plebeium, P. polypodioides var. aciculare, $P$. polypodioides var. polypodioides y P. rhodopleuron el desarrollo del gametofito joven se caracteriza por el establecimiento retardado de la célula meristemática, lo que corresponde al tipo-Drynaria (Figs. 13, 14, 16 y 17). Primero se forma una lámina ancha y espatulada que comienza su desarrollo entre los 15 y 25 días (en este momento los protalos jóvenes están aún desnudos; es decir, sin pelos) (Fig. 18), posteriormente adquieren una forma laminar ovada, diferenciándose una célula meristemática a partir de la cual se define un meristemo pluricelular y se desarrolla un cojinete delgado; hacia los 35-45 días aparecen los primeros pelos y entre los 75-80 días los gametofitos adquieren el aspecto espatulado-cordiforme (Figs. 19 y 21).

En $P$. fraternum y P. lepidotrichum el desarrollo protálico es de tipo Aspidium (Figs. 15, 22), que se caracteriza por la presencia y el desarrollo temprano de pelos. Entre los 18-20 días la célula terminal del filamento germinativo se divide longitudinalmente originando dos, una de las cuales desarrolla un pelo y queda inactiva, mientras que la otra célula se sigue partiendo hasta formar marginalmente una célula meristemática que posteriormente es reemplazada por un meristemo pluricelular (27-28 días), el cual adquiere forma de muesca y se vuelve apical por el crecimiento de las alas del gametofito, dando lugar a un cojinete en posición media. Para los 73 días el protalo maduro es cordiforme y con abundantes pelos unicelulares marginales (Fig. 22).

Fase adulta: Los gametofitos de P. fraternum (70-75 días), P. plebeium (42-46 días), P. polypodioides var. aciculare (40-45 días) y P. rhodopleuron (60-90 días), son espatulado-cordiformes y ocasionalmente cordiforme-reniformes (Figs. 21, 24, 25). Presentan alas simétricas, una zona meristemática central bien definida, formando una escotadura y un cojinete, los márgenes son irregulares y muestran pelos unicelulares secretores y bicelulares no secretores, que se distribuyen tanto en el borde como en la superficie de la lámina (30-37 días). En la parte posterior del gametofito se localizan abundantes rizoides, hialinos o de color pardo claro y sin cloroplastos. 

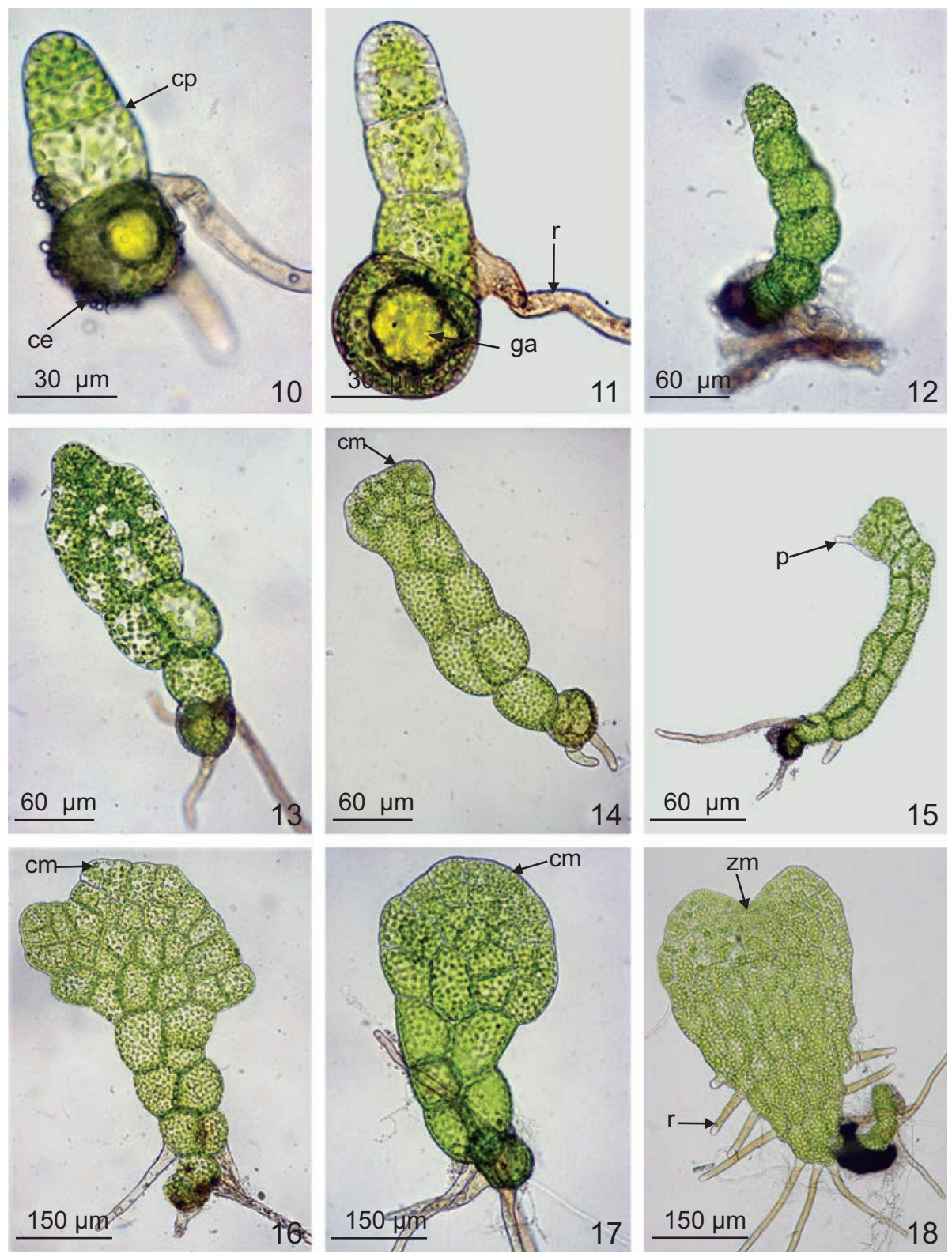

Figs. 10-18. Fases filamentosas y laminares de Polypodium. 10. P. lepidotrichum (20 días), 11. P. rhodopleuron (14 días), 12. P. furfuraceum (22 días), 13-14. P. plebeium (22 días), 15. P. lepidotrichum (20 días), 16. P. furfuraceum (37 días), 17-18. P. polypodioides var. polypodioides (15-20 días), $\mathrm{ce}=$ cubierta de la espora, $\mathrm{cm}=$ célula meristemática, $\mathrm{cp}=$ célula protálica, ga $=$ glóbulo de aceite, $\mathrm{p}=$ pelo, $\mathrm{r}=$ rizoide, $\mathrm{zm}=$ zona meristemática. 
Con respecto a $P$. lepidotrichum (70-75 días), $P$. furfuraceum (70-78 días) y $P$. polypodioides var. polypodioides (27-32 días), sus gametofitos adultos son de forma espatulado-cordiforme a cordiforme (Figs. 20, 26), con pelos unicelulares en el margen (Fig. 22-23); los pelos son abundantes en $P$. lepidotrichum (18-20 días) y $P$. furfuraceum (90-98 días), mientras que en $P$. polypodioides var. polypodioides son escasos (32-40 días).

Gametangios: En $P$. fraternum (70-75 días), $P$. furfuraceum (70-78 días) y $P$. polypodioides var. aciculare (40-45 días), se desarrollaron gametofitos unisexuados femeninos; los arquegonios se localizan ventralmente sobre el cojinete y cerca de la zona meristemática, los cuellos están formados por 4 hileras, cada una con 4 o 5 células de longitud (Figs. 30, 31).

En P. rhodopleuron (100-142 días) se formaron gametofitos masculinos; los anteridios se localizan en la parte marginal de las alas y corresponden al desarrollo y estructura descritos para los gametofitos de los helechos homospóricos leptosporangiados. Están constituidos por tres células: una basal, una media (que rodea a las célula espermatógena) y una opercular (Figs. 32, 33); la liberación de los anterozoides se da mediante la apertura de un poro o a través del desprendimiento de la célula opercular (Fig. 33). Cabe señalar que los gametofitos de esta especie se propagan vegetativamente, pues producen yemas marginales desde etapas laminares jóvenes hasta su estado adulto (80-170 días, Figs. 27-29).

Se observaron gametofitos bisexuados en P. plebeium (42-46 días) y P. polypodioides var. polypodioides (27-32 días). Los anteridios se formaron tanto en la parte media del margen del talo como en la posterior sobre la superficie del mismo cercana a los rizoides, y corresponden al desarrollo y estructura descrito para los helechos homospóricos leptosporangiados. Los arquegonios se localizan ventralmente cerca de la zona meristemática y están constituidos por 4 hileras de células, cada una con 4 o 5 células de longitud (P. plebeium, 63-68 días y en $P$. polypodioides var. polypodioides $40-45$ días).

En las poblaciones de $P$. lepidotrichum se formaron talos unisexuados femeninos (70-75 días) y masculinos de tamaño pequeño (160-170 días), además de que se desarrollaron gametofitos por propagación vegetativa a partir de yemas (150-160 días).

\section{DISCUSIÓN}

Con respecto a las especies estudiadas en este trabajo, cabe señalar que los siete taxa comparten características constantes como tener esporas monoletes y un 

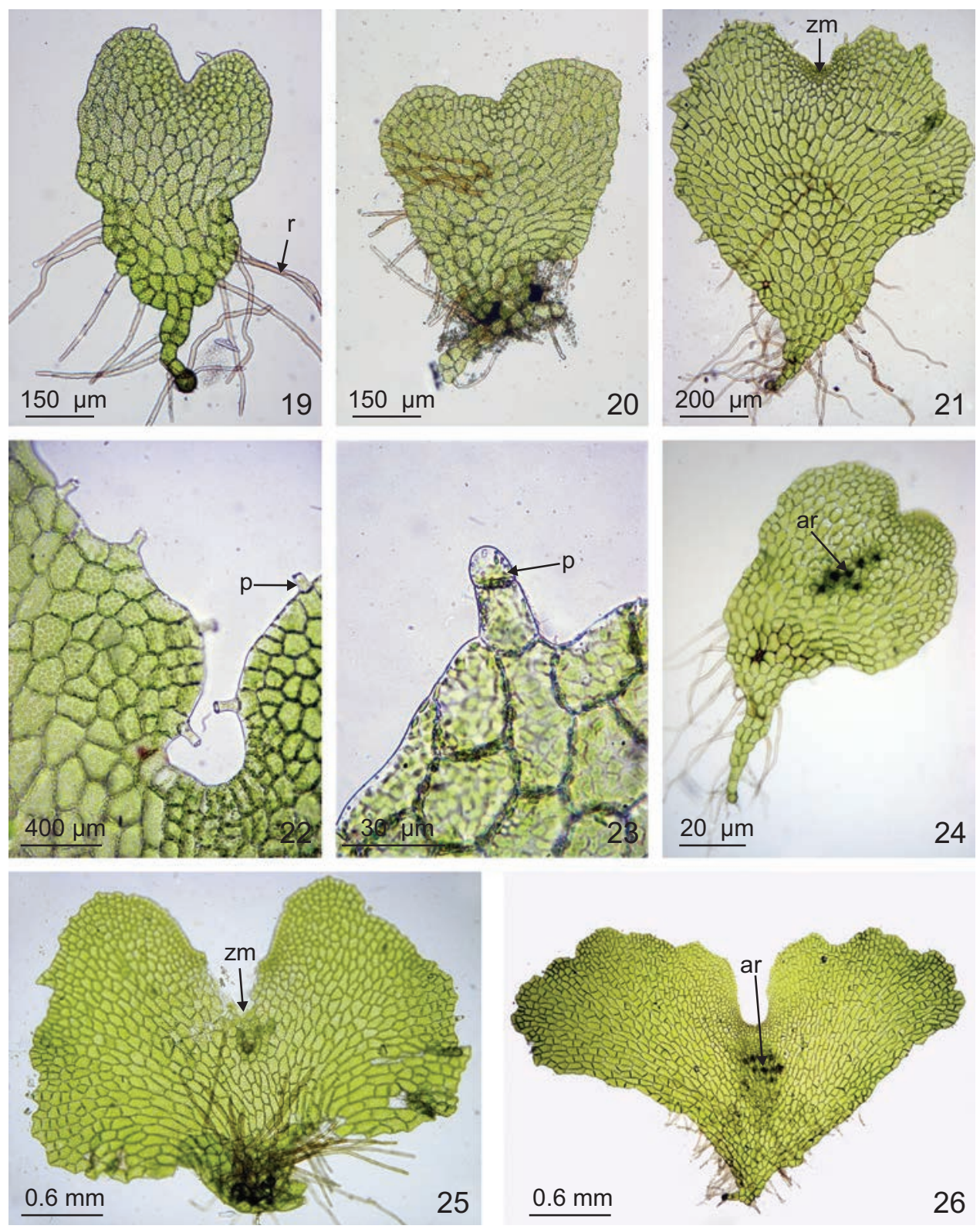

Figs. 19-26. Fases laminares y pelos de Polypodium. 19. P. rhodopleuron (43 días), 20. P. furfuraceum (89 días), 21. P. plebeium (80 días), 22. Zona meristemática con pelos, $P$. fraternum (80 días), 23. Pelo bicelular, P. plebeium (129 días), 24. Gametofito con arquegonios, P. polypodioides var. aciculare (73 días), 25. P. plebeium (73 días), 26. P. lepidotrichum (73 días). ar $=$ arquegonios, $\mathrm{p}=$ pelo, $\mathrm{r}=$ rizoide, $\mathrm{zm}=$ zona meristemática. 
Vázquez-Pérez, et al.: Morfogénesis de la fase sexual de taxa epífitos de Polypodium de México
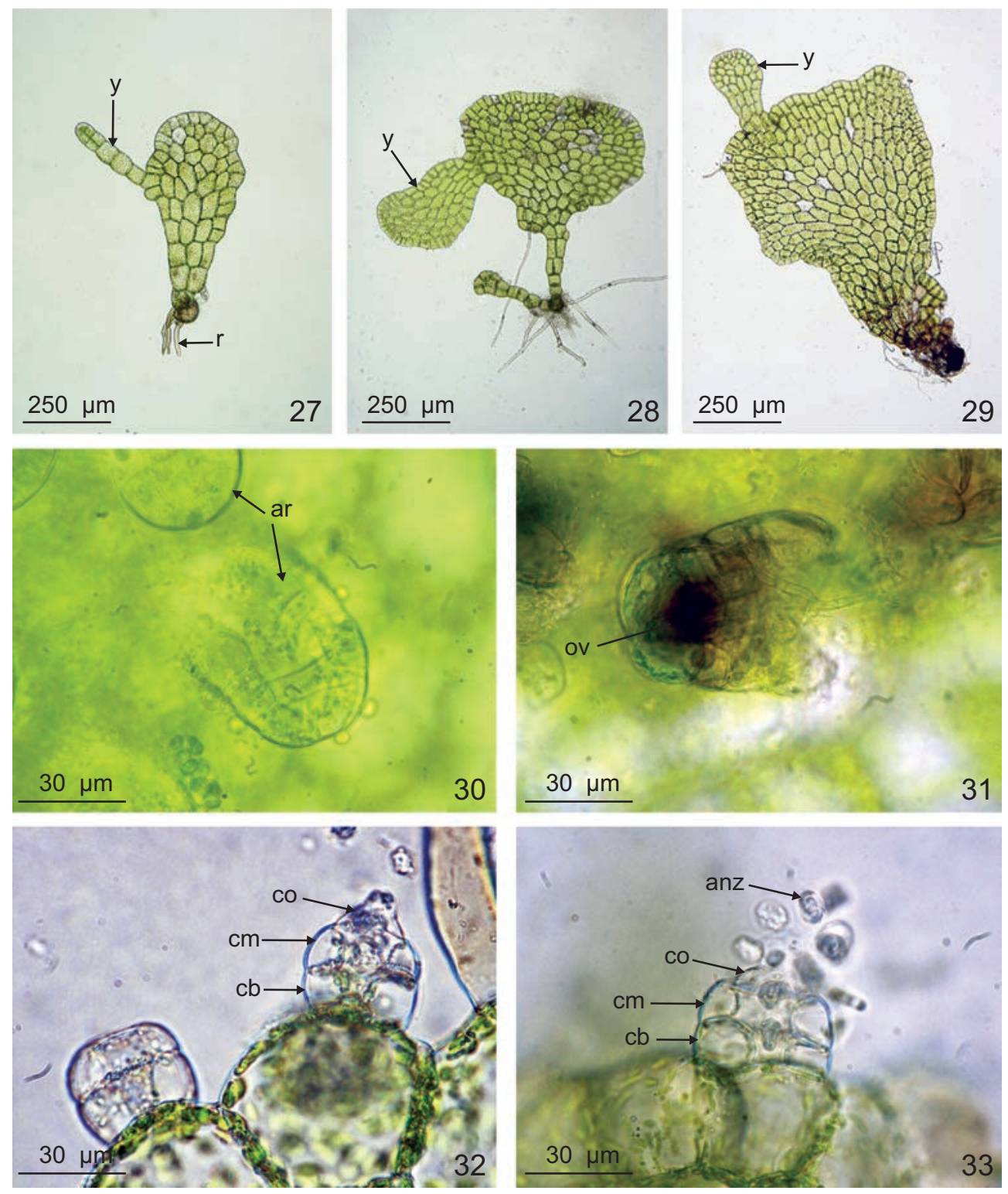

Figs. 27-33. Gametofitos con yemas y gametangios de Polypodium. 27-29. Gametofitos adultos con yemas en distintos estados de desarrollo, P. rhodopleuron (157-164 días), 30. Cuello de arquegonio de $P$. polypodioides var. aciculare (65 días), 31. Cuello de arquegonio de $P$. fraternum (95 días), 32-33. Anteridios de $P$. lepidotrichum (180 días). anz = anterozoide, ar $=$ arquegonios, $\mathrm{cb}=$ célula basal, $\mathrm{cm}=$ célula media, $\mathrm{co}=$ célula opercular, ov = ovocélula, $\mathrm{r}=$ rizoide, $\mathrm{y}=$ yemas. 
perisporio verrucado a tuberculado, con un tamaño promedio de 40 a $75 \mu \mathrm{m}$, siendo las más pequeñas las de Polypodium polypodioides var. aciculare y P. polypodioides var. polypodioides y las más grandes las de P. rhodopleuron (Figs. 1-4), lo que coincide con las características y el intervalo registrados por otros autores para algunas especies de Polypodium (Tryon y Tryon, 1982; Reyes-Jaramillo y PérezGarcía, 1994; Giudice et al., 2004; Gabriel y Galán et al., 2008). En lo que se refiere al patrón de germinación, todos los taxa presentan el tipo Vittaria (6-12 días) y una fase filamentosa que va de 3 a 8 células protálicas. Tanto las características de las esporas como el mencionado modelo coinciden con los señalados por Nayar y Kaur $(1968,1969,1971)$ para las Polypodiaceae.

Se encontraron diferencias en el patrón de desarrollo protálico. En Polypodium furfuraceum, P. plebeium, P. polypodioides var. aciculare, P. polypodioides var. polypodioides y $P$. rhodopleuron se presentó el tipo Drynaria, mientras que en $P$. fraternum y $P$. lepidotrichum fue de tipo Aspidium. En este sentido, es importante resaltar que $P$. fraternum y $P$. rhodopleuron pertenecen a un mismo complejo de especies, el de P. plesiosorum (Moran y Riba, 1995), sin embargo presentan distintos patrones de desarrollo (tipo Aspidium el primero, Drynaria el segundo), lo cual puede ser taxonómicamente significativo sobre todo si consideramos que Tejero-Díez (2005), quien trabajó con este complejo de especies utilizando las características morfológicas y anatómicas del rizoma, de las escamas, de la hoja y de las esporas, concluye que éste constituye un taxon polifilético. P. hispidulum, P. plesiosorum y P. rhodopleuron pertenecen a un grupo, mientras que el resto de las especies, entre las que se encontraría $P$. fraternum, forman parte de otros, lo que coincidiría con la diferencia aquí registrada. En lo que se refiere a $P$. lepidotrichum, que también presenta un desarrollo protálico distinto de los taxa del complejo de especies de $P$. polypodioides que se incluyen en el presente estudio, podemos decir que a diferencia del caso anterior, no se ha realizado aún una revisión taxonómica incluyendo estudios de otros caracteres morfológicos, aunque Reyes-Jaramillo y Pérez-García (1994) habían inferido el mismo tipo de desarrollo protálico en el taxon, razón por la cual se incluyó nuevamente esta especie en el presente estudio. Al respecto, cabe recordar que ya en trabajos, como el de Stokey (1959), se ha propuesto la significancia del desarrollo gametofítico en la delimitación de grupos de taxa, por lo que sería interesante estudiar la morfogénesis de las demás especies que integran a los complejos de $P$. plesiosorum y $P$. polypodioides, para establecer si en este caso hay una relación entre la circunscripción infragenérica y el desarrollo protálico.

Por otro lado se observó una variación en la expresión sexual de los siete taxa, formándose sólo gametofitos femeninos (unisexuados) en P. fraternum, $P$. 
furfuraceum y P. polypodioides var. aciculare; protalos masculinos (unisexuados) y yemas (propagación vegetativa) en $P$. rhodopleuron; gametofitos unisexuales femeninos, masculinos y propagación vegetativa en $P$. lepidotrichum y protalos bisexuales en $P$. plebeium y $P$. polypodioides var. polypodioides. En este sentido, es importante recordar que en los helechos homospóricos se ha registrado la presencia de tres tipos de fecundación, el primero es denominado apareamiento intergametofítico, en el que se produce la fusión de gametos procedentes de distintos gametofitos desarrollados a partir de esporas de diferentes esporofitos de la misma especie; el segundo se conoce como autofecundación intergametofítica en la que se unen gametos de diferentes gametofitos que proceden de esporas del mismo esporofito; y la tercera es la autofecundación intragametofítica, en la que se unen gametos generados en el mismo gametofito (Chiou et al., 2002). Cada uno de estos tipos de fecundación puede relacionarse de manera indirecta con la expresión sexual que se presenta en las distintas especies estudiadas, por ejemplo en los taxa que sólo tienen gametofitos unisexuales ( $P$. fraternum, $P$. furfuraceum, $P$. polypodioides var. aciculare y $P$. rhodopleuron) se ve favorecida la fecundación intergametofítica; mientras que en aquellos en los que se presentan gametofitos femeninos y gametofitos masculinos de menor tamaño (P. lepidotrichum), es probable que se esté produciendo lo que se conoce como efecto anteridiógeno, mediante una sustancia secretada por gametofitos femeninos adultos que promueve la formación de protalos masculinos, incrementándose la probabilidad de que se dé la autofecundación intergametofítica y por último los que desarrollan gametofitos bisexuales (P. plebeium y P. polypodioides var. polypodioides), están llevando a cabo la autofecundación intragametofítica.

La propagación vegetativa a partir de yemas en los gametofitos de especies epífitas se ha relacionado con las características particulares del hábitat, en el que la presencia de otros organismos vegetales (como por ejemplo musgos) hace que las interacciones entre los protalos sean más complejas que en un ambiente terrestre, siendo necesario alargar significativamente la vida activa de la fase sexual hasta que se den las condiciones que permitan la fecundación intergametofítica (Chiou y Farrar, 1997b; Shorina, 2001; Chiou et al., 2002). En este caso, si bien todas las especies estudiadas tienen hábito epífito, en sólo dos de ellas ( $P$. lepidotrichum y $P$. rhodopleuron) sus gametofitos vegetativos formaron yemas.

Son diversos los trabajos en los que se ha señalado que Polypodium, tal como tradicionalmente se define, es polifilético (Haufler y Ranker, 1995 y Schneider et al., 2004), de ahí la importancia de encontrar caracteres alternativos que contribuyan junto con otras fuentes de información, como la morfología del esporofito, la ana- 
tomía, la biología molecular etc., a establecer de manera más clara, los límites del género. Por ello es importante resaltar que la información generada hasta el momento para algunas especies de Polypodium y la obtenida en este estudio, muestran que si bien las características de las esporas y el tipo de germinación son constantes a nivel genérico, no ocurre lo mismo con el desarrollo protálico, lo que pudiera ser de utilidad en la delimitación taxonómica.

Finalmente, si bien son diversos los trabajos en los que se indica la importancia de tener distintos patrones de expresión sexual, en la colonización de nuevos hábitats así como en el mantenimiento de la diversidad genética (Cousens, 1975; Chiou y Farrar, 1997a, 1997b; Shorina, 2001; Wubs et al., 2010), es necesario continuar con trabajos experimentales que nos permitan tratar de entender la dinámica de este proceso, especialmente en los helechos epífitos.

\section{AGRADECIMIENTOS}

Agradecemos a Ricardo Valdez Ávila el apoyo brindado en la parte experimental del trabajo. A Jacqueline Ceja Romero por la revisión crítica del manuscrito, así como por sus comentarios para enriquecer el contenido de este artículo y finalmente a los revisores anónimos por sus valiosas sugerencias.

\section{LITERATURA CITADA}

Agnew, N., A. McCabe y D. L. Smith. 1984. Photocontrol of spore germination in Polypodium vulgare. New Phytol. 96: 167-178.

Agnew, N., A. McCabe y D. L. Smith. 1985. Photocontrol of protonema growth in Polypodium vulgare L. Proc. Roy. Soc. Edinburgh 86: 448-456.

Atkinson, L. R. 1973. The gametophytes and family relationships. In: Jermy, A. C., J. A. Crabbe y B. A. Thomas (eds.). The phylogeny and classification of the ferns. Bot. J. Linn. Soc. (Suppl 1.) 67: 73-90.

Atkinson, L. R. y A. G. Stokey. 1964. Comparative morphology of the gametophyte of homosporous ferns. Phytomorphology 14: 51-70.

Atkinson, L. R. y A. G. Stokey. 1970. Gametophyte of Polypodium chnoodes. Phytomophology 20: 363-367.

Chiou, W. L. y D. R. Farrar. 1997a. Antheridiogen production and response in Polypodiaceae species. Amer. J. Bot. 84: 663-640.

Chiou, W. L. y D. R. Farrar. 1997b. Comparative gametophyte morphology of selected species of the family Polypodiaceae. Amer. Fern J. 87: 77-86. 
Vázquez-Pérez, et al.: Morfogénesis de la fase sexual de taxa epífitos de Polypodium de México

Chiou, W. L., D. R. Farrar y T. A. Ranker. 2002. The mating systems of some epiphytic Polypodiaceae. Amer. Fern J. 92: 65-79.

Cousens, M. L. 1975. Gametophyte sex expression in some species of Dryopteris. Amer. Fern J. 65: 39-42.

Davie, J. H. 1951. The development of the antheridium in the Polypodiaceae. Amer. J. Bot. 38: 621-628.

Davie, S. 1978. Studies on spore morphology of some members of Polypodiaceae. Brenesia 14(15): 283-303.

Gabriel y Galán, J. M., C. Prada y C. Rolleri. 2008. Germinación de la espora, morfología del gametofito y expresión sexual de Polypodium feuillei Bertero (Polypodiaceae). Gayana Bot. 65: 14-22.

Giudice, G. E., M. A. Morbelli, M. R. Piñeiro, M. Copello y G. E. Source. 2004. Spore morphology of the Polypodiaceae from northwestern Argentina. Amer. Fern J. 94: 9-27.

Hartman, M. E. 1931. Anteridial dehiscence in the Polypodiaceae. Bot. Gaz. (Crawfordsville) 91: 252-276.

Haufler, C. H. y T. A. Ranker. 1995. Rbcl sequences provide phylogenetic insights among sister species of the fern genus Polypodium. Amer. Fern J. 85: 361-374.

Klekowski, E. J. 1969. Reproductive biology of the Pteridophyta. III. A study of Blechnaceae. Bot. J. Linn. Soc. 62: 361-377.

Knudson, L. 1940. Permanent changes of chloroplast induced by X rays in the gametophytes of Polypodium aureum. Bot. Gaz. (Crawfordsville) 101: 721-758.

Kott, L. S. y R. L. Peterson. 1974. A comparative study of gametophyte development in the diploid and tetraploid race of Polypodium virginianum. Canad. J. Bot. 52: 91-96.

Mickel, J. T. y A. R. Smith. 2004. The pteridophytes of Mexico. Mem. New York Bot. Gard. 88: $1-1054$.

Moran, R. C. y R. Riba. 1995. Flora Mesoamericana. Vol. 1. Psilotaceae a Salviniaceae. Instituto de Biología, Universidad Nacional Autónoma de México, Missouri Botanical Garden y The Natural History Museum, London. México, D.F. 470 pp.

Muccifora, S. 2008. Effects of copper on spore germination, growth and ultraestructure of Polypodium cambricum (L.) gametophytes. Environm. Pollut. 153: 369-375.

Nayar, B. K. 1955. Studies in Polypodiaceae III. Loxogramme. J. Indian Bot. Soc. 34: 396407.

Nayar, B. K. 1962. Morphology of spores and prothalli of some species of Polypodiaceae. Bot. Gaz. (Crawfordsville) 123: 223-232.

Nayar, B. K. y P. Kachroo. 1953. Studies in Polypodiaceae I- Contributions to the morphology of Drynaria Bory, D. quercifolia (L.) J. Sm. and D. propinqua (Wall.) J. Sm. Phytomorphology 3: 411-424.

Nayar, B. K. y S. Kaur. 1968. Spore germination in homosporous ferns. J. Palynology 4: 1-14.

Nayar, B. K. y S. Kaur. 1969. Types of prothallial development in homosporous ferns. Phytomorphology 19: 179-188.

Nayar, B. K. y S. Kaur. 1971. Gametophytes of homosporous ferns. Bot. Rev. (Lancaster) 37: 295- 396. 
Nayar, B. K. y F. Raza. 1970. Morphology of the prothalli of some species of the Polypodiaceae - II: Lepisorus loriformis, L. thunbergianus, Polypodium vulgare and Weatherbya accedens. J. Indian Bot. Soc. 49: 81-86.

Pérez-García, B., A. Mendoza, R. Riba y L. D. Gómez-Pignataro. 2001. Development of the sexual phase of Pseudocolysis bradeorum (Polypodiaceae). Amer. Fern J. 91: 214-226.

Pickett, F. L. y L. A. Thayer. 1927. The gametophytic development of certain ferns: Polypodium vulgare var. occidentale and Pellaea densa. Bull. Torrey Bot. Club 54: 249-255.

Raghavan, V. 1974. Control of differentiation in the fern gametophyte. Amer. Sci. 62: 465475.

Raghavan, V. 1980. Cytology, physiology and biochemistry of germination of fern spores. Int. Rev. Cytol. 62: 69-118.

Raghavan, V. 1985. Cytology of germination of spores of homosporous ferns. Proc. Roy. Soc. Edinburgh B 86: 5-12.

Raghavan, V. 1989. Developmental biology of ferns gametophytes. Cambridge University Press. Cambridge. 361 pp.

Ramírez, M. R. y B. Pérez-García. 1998. Fase gametofítica del helecho Microgramma nítida (Polypodiaceae). Rev. Biol. Trop. 46(3): 587-593.

Ranal, M. A. 1991. Germination of Polypodium hirsutissimum spores and antheridia formation in darkness. Rev. Brasil. Biol. 51: 675-679.

Reixach, N. J., J. C. Irrure-Santilari, E. Mele, J. Messeguer y F. Camps. 1996. Biosynthesis of ecdysteroids in vitro prothalli cultures of Polypodium vulgare. Phytochemistry 43: 597-602.

Reyes-Jaramillo, I. y B. Pérez-García. 1994. Morfología y estrategias reproductivas de Polypodium lepidotrichum (Fee) Maxon (Polypodiaceae). Acta Bot. Mex. 28: 71-78.

Reyes-Jaramillo, I., B. Pérez-García y A. Mendoza-Ruiz. 2003. Morfogénesis de los gametofitos de especies mexicanas de Pleopeltis (Polypodiaceae, subfamilia Pleopeltoideae). Rev. Biol. Trop. 51(2): 321-332.

Roy, S. K. 1987. Inhibitory effect of licorine on spore germination and gametophyte development of Polypodium verrucosum Wall. Curr. Sci. 56: 423-425.

Schneider, H., A. R. Smith, R. Cranfill, T. E. Hildebrand, C. H. Haufler y T. A. Ranker. 2004. Unraveling the phylogeny of polygrammoid ferns (Polypodiaceae and Grammitidaceae): exploring aspects of the diversification of epiphytic plants. Molec. Phylog. Evol. 31: 1041-1063.

Shorina, N. I. 2001. Population biology of gametophytes in homosporous Polypodiophyta. Russ. J. Ecol. 32: 164-169.

Smith, A. R., K. M. Pryer, E. Schuettpelz, P. Korall, H. Schneider y P. G. Wolf. 2006. A classification for extant ferns. Taxon 55(3): 705-731.

Smith D. L. 1972. Staining and osmotic propierties of young gametophytes of Polypodium vulgare L. and their bearing on rhizoid function. Protoplasma 74: 465-479.

Smith, D. L. y P. M. Robinson. 1969. The effects of fungi on morphogenesis of gametophytes of Polypodium vulgare L. New Phytol. 68: 113-122.

Smith, D. L. y P. M. Robinson. 1971. Growth factors produced by germinating spores of Polypodium vulgare L. New Phytol. 70: 1043-1052. 
Smith, D. L. y P. M. Robinson. 1975. The effects of spore age on germination and gametophyte development in Polypodium vulgare L. New Phytol. 74: 101-108.

Smith, D. L. y P. G. Rogan. 1970. The effects of population density on gametophyte morphogenesis in Polypodium vulgare L. New Phytol. 69: 1039-1051.

Smith, D. L., P. M. Robinson y R. N. Govier. 1973. Growth factors by gametopytes of Polypodium vulgare L. grown under red and blue light. New Phytol. 72: 1261-1268.

Spies, L. D. y W. G. Krouk. 1977. Photocontrol of germination of spores of the fern Polypodium aureum. Bot. Gaz. (Crawfordsville) 138: 428-433.

Steil, W. N. 1921. The development of prothallia and antheridia from the sex organs of Polypodium iridiodes. Bull. Torrey Bot. Club. 48: 271-284.

Stokey, A. G. 1951. The contribution by the gametophyte to classification of the homosporous ferns. Phytomorphology 1: 34-58.

Stokey, A. G. 1959. Polypodium pectinatum and P. plumula - Polypodiaceae or Grammitidiaceae. Amer. Fern J. 49: 142-146.

Tejero-Díez, J. D. 2005. Revisión taxonómica del complejo Polypodium plesiosorum Kunze (Polypodiaceae, Polypodiophyta). Tesis doctoral. Universidad Autónoma Metropolitana-Iztapalapa. México, D.F. 129 pp.

Tryon, R. M. y A. F. Tryon. 1982. Ferns and allied plants with special reference to tropical America. Springer-Verlag. Nueva York. pp. 715-722.

van Uffelen, G. A. 1997. The spore wall in Polypodiaceae, development and evolution. In: Johns, R. J. (ed.). Holttum Memoral Volume. Royal Botanic Gardens. Kew. pp. 95118.

Welling, C. B. y C. H. Haufler. 1993. Antheridiogen and its puzzling effects on Polypodium australe. Amer. J. Bot. 80 (Supplement): 113-121.

Wubs, E. R., G. A. de Groot, H. J. During, J. C. Vogel, M. Grundmann, P. Bremer y H. Schneider. 2010. Mixed mating system in the fern Asplenium scolopendrium: implications for colonization potential. Ann. Bot. 106: 583-590.

Wuist, E. D. 1916. Branched prothallia in the Polypodiaceae. Bull. Torrey Bot. Club 43: 365-383. 
